\title{
Biotinylation of optically responsive gold/polyelectrolyte nanostructures
}

\author{
Sónia O. Pereira • Tito Trindade • Ana Barros-Timmons
}

Published online: 14 December 2014

(C) The Author(s) 2014. This article is published with open access at SpringerLink.com

\begin{abstract}
The development of analytical platforms incorporating Au nanoparticles (NPs) for optical biosensing requires approaches to produce optically responsive and target selective materials. In the present work, fluorescence emission and bioselectivity of Au NPs have been combined by a one-step surface functionalization using fluorescein isothiocyanate (FITC) and biotin. First, colloidal Au NPs $(d=17.7 \pm 2.7 \mathrm{~nm})$ exhibiting an absorption maximum $\left(\lambda_{\max }\right)$ at $524.5 \mathrm{~nm}$ have been surface-modified with the polyelectrolytes (PE) poly(allylamine hydrochloride) (PAH) and poly(sodium styrene sulfonate) (PSS). Two strategies were then assessed for the functionalization of Au NPs: (i) functionalization of PAH with biotin and subsequent coating of the NPs and (ii) surface modification of the NPs with the PE followed by postfunctionalization with biotin. The effect of the surface functionalization on the colloidal NPs was monitored by optical absorption and fluorescence emission spectroscopies, dynamic light scattering (DLS), zeta potential measurements and transmission electron microscopy (TEM). Finally, the optical response and specificity of the functionalized NPs towards avidin was evaluated by optical measurements. The results have shown that these hybrid nanostructures have potential to be used in biosensing. In particular, the aggregation of biotinylated $\mathrm{Au}$ assemblies associated with the biorecognition of avidin could be assessed by fluorescence emission spectroscopy.
\end{abstract}

Electronic supplementary material The online version of this article (doi:10.1007/s13404-014-0153-x) contains supplementary material, which is available to authorized users.

S. O. Pereira $\cdot$ T. Trindade $\cdot$ A. Barros-Timmons $(\bowtie)$

Department of Chemistry-CICECO, University of Aveiro,

Aveiro 3810-193, Portugal

e-mail: anabarros@ua.pt
Keywords Biosensor · Gold nanoparticles · Optical properties · Hybrid nanostructures · Polyelectrolytes · Biotinylation

\section{Introduction}

Gold nanoparticles (Au NPs) have been widely studied for diverse bioapplications namely due to their optical properties which are dependent on the morphological characteristics and surrounding medium [1-5]. These properties result from the fact that colloidal Au NPs show a surface plasmon resonance (SPR) absorption band in the visible region whose location and profile depends on particle size and shape, refractive index of the surrounding medium and interparticle neighbouring [4]. Yet, the application of Au NPs in biological systems requires further surface modification to achieve stability in aqueous medium and the ability to interact with the surroundings, thus enabling the detection of target bioanalytes. Indeed, functionalized Au NPs can be used as a platform for bioanalytes-receptor interactions which can be detected by optical means as a result of changes of interparticles distance (e.g. aggregation) [6, 7]. Examples of the use of this property include the recognition of a specific DNA strand $[8,9]$ as well as protein-ligand systems such as (strept)avidinbiotin $[10,11]$.

There are several methods to synthesize Au NPs which can result in different sizes and shapes of NPs [6]. A popular method is the citrate method reported by Turkevich et al. [12] which yields nanospheres with diameters between $\sim 10$ and $50 \mathrm{~nm}$ stabilized by citrate anions. These Au NPs can be further functionalized in order to ensure adequate stabilization in other media. For instance, Au NPs can be modified using Lewis bases, thiols [13-15], and DMAP (4-(dimethylamino)pyridine) which provide an organic coat at the surface [16]. In fact, the surface chemistry of NPs is an 
area of major interest due to the possibility of controlling the shell composition, charge, thickness, and the ability for surface functionalization using diverse strategies that include attachment of antibodies, fluorophores, and polymers. As a consequence, these functionalized $\mathrm{Au}$ NPs have found wide interest in areas such as pharmaceutics, biomedicine, photonics, and new optical materials [17-22].

Another way to obtain stable colloids is by coating the NPs via electrostatic assembly of polyelectrolytes following the layer-by-layer (LbL) deposition method [14, 23-25]. This type of coating not only stabilizes the nanoparticles, but also provides a path to modify the surface with molecules of interest in bioapplications [26-28]. It is a very simple, easy, and versatile method, however, to yield stable coated nanoparticles an efficient separation of nanoparticles after the coating must be ensured [17, 24, 27, 29]. This is not straightforward especially when multifunctional systems are involved.

Another effect associated with the use of colloidal Au NPs that can be exploited in optical biosensing results from the fluorescence emission quenching of a neighbouring fluorophore. This effect occurs when the emission spectrum of a fluorescent species (donor) overlaps the SPR band of $\mathrm{Au}$ NPs (acceptor). In close proximity, the donor molecules transfer energy to the acceptor particle resulting in fluorescence quenching. This has been described as the result of a combination of two effects: FRET (fluorescence resonance energy transfer) and NSET (nanoparticle surface energy transfer), the latter occurring for distances nearly twice as those observed for FRET [30-34]. Schneider et al. [35] have shown the dependence of the distance between Au NPs and two fluorophores (fluorescein isothiocyanate-FITC and Lissamine Rhodamine-LISS) on the quenching effect. The study of this effect allows monitoring the binding and unbinding states of the analyte because they are associated to changes in fluorescence intensity [36-39]. In turn, Aslan et al. [40, 41] have investigated the fluorescence quenching effect using biotinylated Au NPs and anti-biotin labelled with the fluorophore Alexa ${ }^{\mathrm{TM}}$ 488. These authors have observed that after addition of biotinylated Au NPs to an anti-biotin solution, there was a strong decrease of the fluorophore emission intensity, which increased again upon further addition of free biotin.

The present work focuses on the surface modification and functionalization of Au NPs using polyelectrolytes and the electrostatic self-assembly method to confer fluorescence and biospecificity to the resulting colloids. As such, a variety of procedures have been investigated that combine the use of an organic dye (FITC) and a specific bioanalyte (biotin). The ensuing functionalized $\mathrm{Au} / \mathrm{PE}$ assemblies were used in biosensing experiments to explore their optical properties as quencher when aggregation of Au NPs was induced.

\section{Materials and methods}

\section{Chemicals}

Hydrogen tetrachloroaurate (III) trihydrate, 99.9+\% $\left(\mathrm{HAuCl}_{4} \cdot 3 \mathrm{H}_{2} \mathrm{O}\right)$, sodium citrate tribasic dehydrate, $\geq 99 \%$ $\left(\mathrm{Na}_{3} \mathrm{C}_{6} \mathrm{H}_{5} \mathrm{O}_{7} \cdot 2 \mathrm{H}_{2} \mathrm{O}\right)$, biotin, $\geq 99 \%$, avidin from egg white $\geq$ $98 \%$, fluorescein isothiocyanate isomer I (FITC), N-(3dimethylaminopropyl)-N'-ethyl-carboiimide (EDC), poly(allylamine hydrochloride) MW 15,000 (PAH), and poly(styrenesulfonic acid sodium salt) MW 17,000 (PSS) were purchased from Sigma-Aldrich. Potassium hydroxide (pellets) was purchased from LabChem. Dimethyl sulfoxide (DMSO), $>99.7 \%$, was purchased from SdS Carlo Erba. Ultrapure water purified using a Station 8000/Station 9000 purification unit was used throughout the work.

\section{Instrumentation}

The UV/VIS spectra of the colloids were recorded using quartz cells and a Jasco V-560 UV/VIS spectrometer, using water as the reference. The fluorescence spectra of the colloids were recorded using quartz cells and a FluoroMax-3 Horiba Jobin Yvon spectrometer. Transmission electron microscopy (TEM) images were obtained using a Hitachi H-9000 microscope operated at $300 \mathrm{kV}$. The samples were prepared by placing a drop of diluted colloidal solutions on a copper grid coated with an amorphous carbon film and left to evaporate. The zeta potential and dynamic light scattering (DLS) measurements were carried out using a ZetaSizer Nano ZS Model Zen 3500 from Malvern. For DLS measurements, one drop of the colloidal solution was diluted in circa $1 \mathrm{~mL}$ of water. For the zeta potential measurements, the colloids were analysed as prepared (the $\mathrm{pH}$ of the colloids varied between 5.5 and 6.5, room temperature). Centrifugation was performed in a Force 1618 Microcentrifuge at room temperature.

\section{Synthesis of Au NPs}

Ten milliliter of sodium citrate solution $(38.8 \mathrm{mM})$ were added to $100 \mathrm{~mL}$ of $\mathrm{HAuCl}_{4} \cdot 3 \mathrm{H}_{2} \mathrm{O}$ solution $(1 \mathrm{mM})$ previously brought to $90{ }^{\circ} \mathrm{C}$ and under vigorous stirring. After $1 \mathrm{~h}$, heating was switched off and stirring was kept overnight. The concentration of the gold nanoparticle stock solution obtained was estimated according to the method published by Schneider et al. [35] to be around $12 \mathrm{nmol} / \mathrm{L}$ in particles. This solution was centrifuged in $1.5 \mathrm{~mL}$ Eppendorfs for $1.5 \mathrm{~h}$ at $14,000 \mathrm{rpm}(15,996 \mathrm{~g})$ before use. The supernatant was removed from each tube and replaced by ultrapure water using a volume twice that of the initial.

In order to prepare the poly(allylamine hydrochloride) polyelectrolyte modified with fluorescein (PAHF), PAH (208.6 mg) was dissolved in $20 \mathrm{~mL}$ of water and aqueous 
$\mathrm{KOH}$ solution $(3 \mathrm{M}$ ) was added dropwise to the stirred solution until $\mathrm{pH}$ 9.4. Next, fluorescein isothiocyanate $(8.4 \mathrm{mg})$ was added, and the solution was stirred overnight under exclusion of light. Under the same conditions, the mixture was dialysed (Spectra/Por ${ }^{\circledR}$ Dialysis Membrane MWCO 6$8 \mathrm{kDa}$ from SpectrumLabs.com) using DMSO in water $(\sim 0.1$ $\% \mathrm{~V} / \mathrm{V}$ ) to remove unreacted fluorophore. During this process, the dialysate was monitored by optical spectroscopy.

Biotinylated PAH (PAHB) and PAHF (PAHFB) polyelectrolytes were prepared by adding $2 \mathrm{~mL}$ of biotin solution $(1 \mathrm{mg} / \mathrm{mL})$ and $2 \mathrm{~mL}$ of EDC solution $(2 \mathrm{mg} / \mathrm{mL})$ to $20 \mathrm{~mL}$ of a PAH solution $(5 \mathrm{mg} / \mathrm{mL}, \mathrm{pH}=5.5)$ or PAHF $(\sim 5 \mathrm{mg} / \mathrm{mL}$, $\mathrm{pH}=5.5)$. The solutions were mixed and stirred for $1 \mathrm{~h}$ at room temperature. These solutions were not dialyzed and were used to modify Au NPs.

Preparation of $\mathrm{Au} / \mathrm{PE}$ hybrid nanostructures

In this work, $\mathrm{Au} / \mathrm{PE}$ hybrid nanostructures with a distinct number of layers ( $n=0$ or 2 ) have been prepared: $\mathrm{Au}_{0} \mathrm{PAH}$ (with a single $\mathrm{PE}$ layer) and $\mathrm{Au}_{2} \mathrm{PAH}$ (with one intermediary bi-layer of PAH/PSS and a third outermost layer of PE), as illustrated in Fig. 1.

Typically, $60 \mathrm{~mL}$ of the Au NPs colloid was added dropwise, under vigorous stirring, to $60 \mathrm{~mL}$ of a solution of PAH $(5 \mathrm{mg} / \mathrm{mL})$ and stirred for $2 \mathrm{~h}$, at room temperature. The mixture was centrifuged for $60 \mathrm{~min}$ at 14,000 rpm (15,996 g). The supernatant was removed and the precipitate re-dispersed in water. This step was repeated once again and the redispersed volume after the second centrifugation was adjusted in order to obtain a final volume of $60 \mathrm{~mL}$ to yield a NPs colloid designated as $\mathrm{Au}_{0} \mathrm{PAH}$. Then, these NPs were added dropwise, under vigorous stirring, to $60 \mathrm{~mL}$ of a solution of PSS $(6.25 \mathrm{mg} / \mathrm{mL})$ and stirred for $2 \mathrm{~h}$ at room temperature. The mixture was centrifuged for $60 \mathrm{~min}$ at $14,000 \mathrm{rpm}(15$,
$996 \mathrm{~g}$ ). The supernatant was removed and the precipitate redispersed in water. This centrifugation process was repeated and the re-dispersed volume after this second centrifugation was adjusted in order to obtain a final volume of $60 \mathrm{~mL}$. At the end of this second surface modification, the hybrid nanostructures $\mathrm{Au} / \mathrm{PAH} / \mathrm{PSS}$ named as $\mathrm{Au}_{2}$ NPs have been obtained (Fig. 1b). Finally, the outermost layer (Fig. 1c) was deposited following the same procedure and using two biotinylation strategies.

\section{Biotinylation-strategy 1}

The biotinylation of the surface-modified Au NPs was first explored following a procedure similar to the preparation of $\mathrm{Au}_{0}$ and $\mathrm{Au}_{2}$ NPs. The modified polyelectrolytes-PAHF, PAHB or PAHFB have been used in this strategy using $10 \mathrm{~mL}$ of colloid. The scheme illustrating this surface modification is shown in Fig. 1c.

\section{A posteriori biotinylation-strategy 2}

A second strategy for the biotinylation of the Au NPs was also investigated following the path also illustrated in Fig. 1c. In this procedure, $1 \mathrm{~mL}$ of biotin $(1 \mathrm{mg} / \mathrm{mL})$ and $1 \mathrm{~mL}$ of EDC $(2 \mathrm{mg} / \mathrm{mL})$ were added to the colloids $\mathrm{Au}_{\mathrm{n}} \mathrm{PAH}$ or $\mathrm{Au}_{\mathrm{n}} \mathrm{PAHF}(10 \mathrm{~mL})$. The mixtures were stirred for $1 \mathrm{~h}$ and then centrifuged (60 min, 14, $000 \mathrm{rpm}-15,996 \mathrm{~g}$ ) to remove the unreacted biotin. The supernatant was removed and replaced by water (final volume $=10 \mathrm{~mL}$ ). The resulting nanostructures were named $\mathrm{Au}_{n} \mathrm{PAH}+\mathrm{B}$ and $\mathrm{Au}_{\mathrm{n}} \mathrm{PAHF}+\mathrm{B}$, respectively. The "+" means that biotinylation was carried out a posteriori, thus yielding samples distinct from those obtained in strategy 1 .

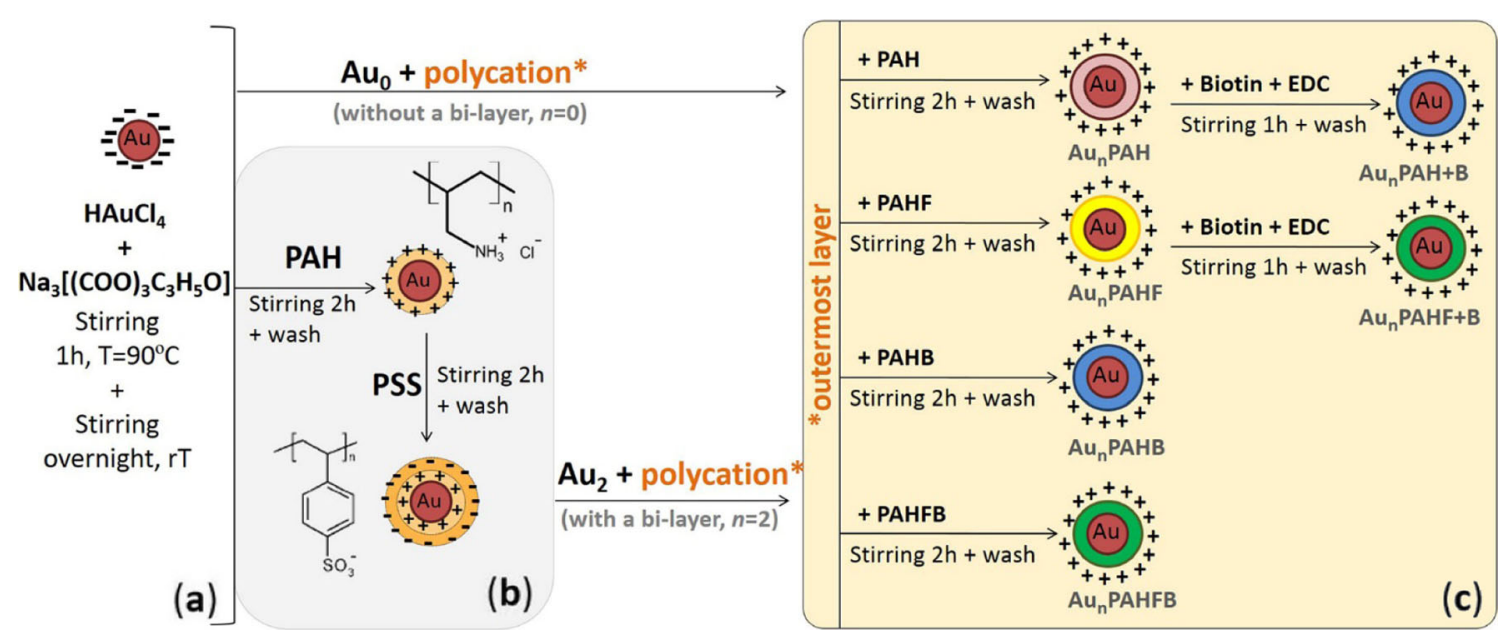

Fig. 1 Preparation of Au/PE hybrid nanostructures. a Synthesis of Au NPs. b Functionalization of Au/Citrate NPs with poly(allylamine hydrochloride) (PAH) and poly(styrenesulfonic acid sodium salt) (PSS). $\mathbf{c}$ Functionalization of $\mathrm{Au}_{\mathrm{n}}$ with the outermost layer 
Optical response of $\mathrm{Au} / \mathrm{PE}$ assemblies to avidin

In order to evaluate the potential use of the biotinylated hybrid $\mathrm{Au} / \mathrm{PE}$ nanostructures in optical biosensing, the optical behavior of the respective colloids was studied in the presence of avidin. In this case, the samples $\mathrm{Au}_{\mathrm{n}} \mathrm{PAH}$ and $\mathrm{Au}_{\mathrm{n}} \mathrm{PAHF}$ were used as blanks regarding the response to avidin. One hundred microliter of an avidin solution $(0.4 \mathrm{mg} / \mathrm{mL})$ was added to $1 \mathrm{~mL}$ of the colloidal solution diluted twofold. One week later, optical measurements have also been performed in order to evaluate the stability of the colloids. Parallel to this, as it was thought that the amount of avidin added could be too small to trigger a specific response, the theoretical quantity of avidin to interact with all the biotin in the system was added to each colloid. The spectra were recorded immediately upon addition of the avidin and $2 \mathrm{~h}$ later. For adequate evaluation of the optical response of the colloids in the presence of avidin, data were compiled as described below.

The original colloidal Au NPs presented a SPR band at around $525 \mathrm{~nm}$. The intensity of this band decreased, and light absorption between 600 and $750 \mathrm{~nm}$ became noticeable due to particle aggregation. In a first approximation, by assuming that these differences in terms of band intensity are mainly due to an aggregation process and by not taking into account band broadening, we have defined a semi-quantitative parameter indicative of aggregation extension due to the presence of avidin:

$$
\begin{aligned}
& \Delta \mathrm{A}(\text { avidin } / \text { colloid }) \\
& =\frac{A_{\mathrm{SPR}}(\text { colloid }+ \text { avidin })-A_{650}(\text { colloid }+ \text { avidin })}{A_{\mathrm{SPR}}(\text { colloid })-A_{650}(\text { colloid })}
\end{aligned}
$$

where $A_{\text {SPR }}$ (colloid + avidin): absorbance at the SPR wavelength for a colloid in the presence of avidin, $A_{650}$ (colloid + avidin): absorbance at $650 \mathrm{~nm}$ for a colloid in the presence of avidin, $A_{\mathrm{SPR}}$ (colloid): absorbance at the SPR wavelength for the initial colloid and $A_{650}$ (colloid): absorbance at $650 \mathrm{~nm}$ for the initial colloid.

For the FITC functionalized Au colloids, attempts have been made to correlate fluorescence measurements with aggregation effects due to the presence of avidin. Hence, the ratio between the fluorescence intensity (at $517 \mathrm{~nm}$ ) of the $\mathrm{Au}$ colloid in the presence of avidin and that of the initial $\mathrm{Au}$ colloid, $I_{517}$ (avidin) $/ I_{517}$ (colloid), was calculated for the experimental conditions indicated in the respective graphs.

\section{Results and discussion}

The preparation of stable Au hydrosols that allows their efficient separation and manipulation without compromising their optical properties is not an easy task. This is particularly more complex for systems envisaging multifunctionality that associate other properties to the plasmonic behaviour of $\mathrm{Au}$ colloids, as recently reviewed [27]. As such, a systematic strategy was followed in the present work in order to investigate the following aspects: (i) number of polyelectrolyte (PE) layers; (ii) strategy to functionalize the outermost layer of the Au NPs; (iii) adequate optical technique to monitor the biosensing ability of the ensuing functionalized nanostructures. Figure 1 shows the strategy followed in this work towards the biotinylation of $\mathrm{Au} / \mathrm{PE}$ assemblies.

The colloidal stability of the functionalized Au nanostructures was monitored by UV/VIS spectroscopy (Fig. 2a), and the spectra collected for all the samples have shown the typical SPR band of colloidal Au, peaked at about $525 \mathrm{~nm}$. The small shift of the SPR maximum from 524.5 to $526.5 \mathrm{~nm}$ after PE treatment of the Au NPs is attributed to slight changes of the dielectric constant of the particles surrounding medium [29]. Also noticeable is the fact that the $\mathrm{Au} / \mathrm{PE}$ nanostructures containing PSS exhibit a strong optical absorption in the UV region (peaked at $225 \mathrm{~nm}$ ) which is due to $\pi-\pi^{*}$ electronic
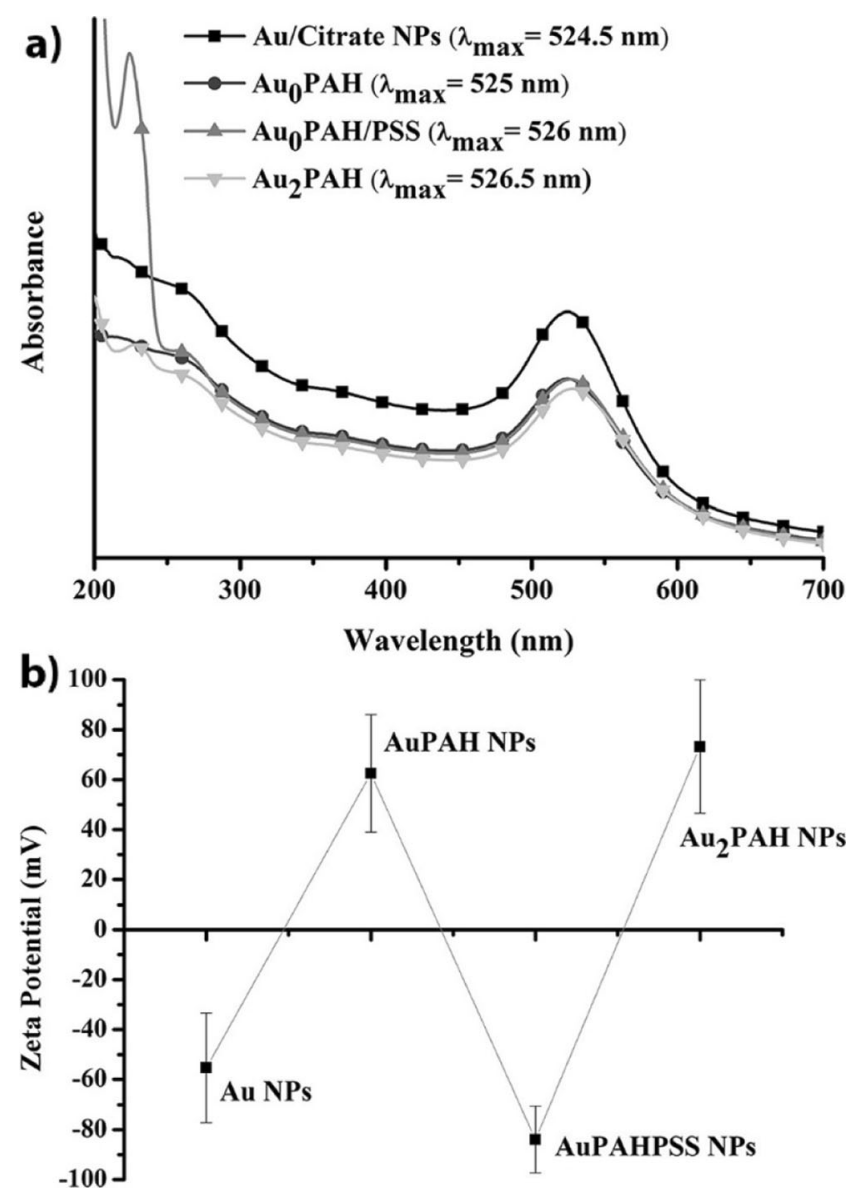

Fig. 2 a UV/VIS spectra of $A_{0} \mathrm{PAH}$ and $\mathrm{Au}_{2} \mathrm{PAH}$ nanostructures and $\mathbf{b}$ zeta potential of $\mathrm{Au} / \mathrm{Citrate}$ colloids and after their treatment with $\mathrm{PAH}$ and PSS (straight lines are to guide the eye) 
transitions of the benzene ring in the polyelectrolyte [29]. The zeta potential measurements (Fig. 2b) performed on colloidal $\mathrm{Au}$ treated with oppositely charged polyelectrolytes agree with the results obtained by UV/VIS spectroscopy. Whilst, the starting Au NPs show a negative $\zeta$ potential $(-55 \mathrm{mV})$ due to the citrate anions adsorbed at their surfaces, upon surface modification with the cationic polyelectrolyte $\mathrm{PAH}$ it changes to $+62 \mathrm{mV}$, at the same $\mathrm{pH}$ value. Conversely, the treatment of the resulting colloids with the anionic polyelectrolyte PSS led to a negative value of zeta potential $(-84 \mathrm{mV})$, and finally, the deposition of the third layer of PAH confers the colloidal particles a highly positive surface charge $(+73 \mathrm{mV})$, that renders colloidal stability to the $\mathrm{Au} / \mathrm{PE}$ dispersed in water.

The morphologies of the Au/PE particles during the functionalization process were probed by TEM, and a systematic analysis of a number of TEM images has shown Au/PE core/shell structures whose cores have an average diameter of 17.7 $\pm 2.7 \mathrm{~nm}$ that did not change significantly after PE treatment. On the other hand, as illustrated in Fig. 3, the TEM images suggest an increase of the shell thickness from $\mathrm{Au}_{0} \mathrm{PAH}$ to $\mathrm{Au}_{2} \mathrm{PAH}$ in line with the increase in the number of PE layers deposited onto the Au cores. From these results, the PE shell thickness was estimated to be about 2-4 nm, which is close to the value reported previously of $1.5 \mathrm{~nm}$ thickness for PAH/PSS bi-layers coating colloidal Au NPs [35]. Figure 3 also shows a set of three Au NPs wrapped by PAH layers, but it must be emphasised that this type of clustered structures was not predominant in the several samples analysed. Instead, the surface-modified $\mathrm{Au}$ samples appeared mainly as discrete particles coated with PE.

The biotinylation of the Au/PE nanostructures was monitored by optical absorption and fluorescence emission measurements for the diverse strategies employed. In general, the visible spectra have shown that biotinylation did not lead to significant changes of the SPR band for the surface-modified samples, namely by comparison with the starting colloid shown in Fig. 2a. However, an exception was observed for the $\mathrm{Au}_{0} \mathrm{PAH}+\mathrm{B}$ sample, in which the biotinylation of $\mathrm{Au}_{0} \mathrm{PAH}$ resulted in a colloid whose visible spectrum also shows an absorption band peaked at $650 \mathrm{~nm}$ (Fig. 4a). Even though this effect is slightly detected for sample $\mathrm{Au}_{0} \mathrm{PAHB}$, it is hardly significant. This new band is probably associated with interparticle plasmon coupling resulting from the loss of colloidal stability during the biotinylation of $\mathrm{Au}_{0} \mathrm{PAH}$ colloids. Interestingly, the nanostructures having an extra PE bilayer $\left(\mathrm{Au}_{2} \mathrm{PAH}+\mathrm{B}\right)$ did not show such optical behaviour upon a similar biotinylation treatment, as illustrated in Fig. 4b. Although there is a slight red shift of the SPR band $(\Delta \lambda=2.5 \mathrm{~nm})$ observed in the spectra of the $\mathrm{Au}_{2} \mathrm{PAH}$ colloid and of the corresponding biotinylated sample $\left(\mathrm{Au}_{2} \mathrm{PAH}+\mathrm{B}\right)$, both visible spectra are similar. These results indicate that the intermediate PAH/PSS coating confers more robustness towards a posteriori treatment with biotin (or post-biotinylation), as
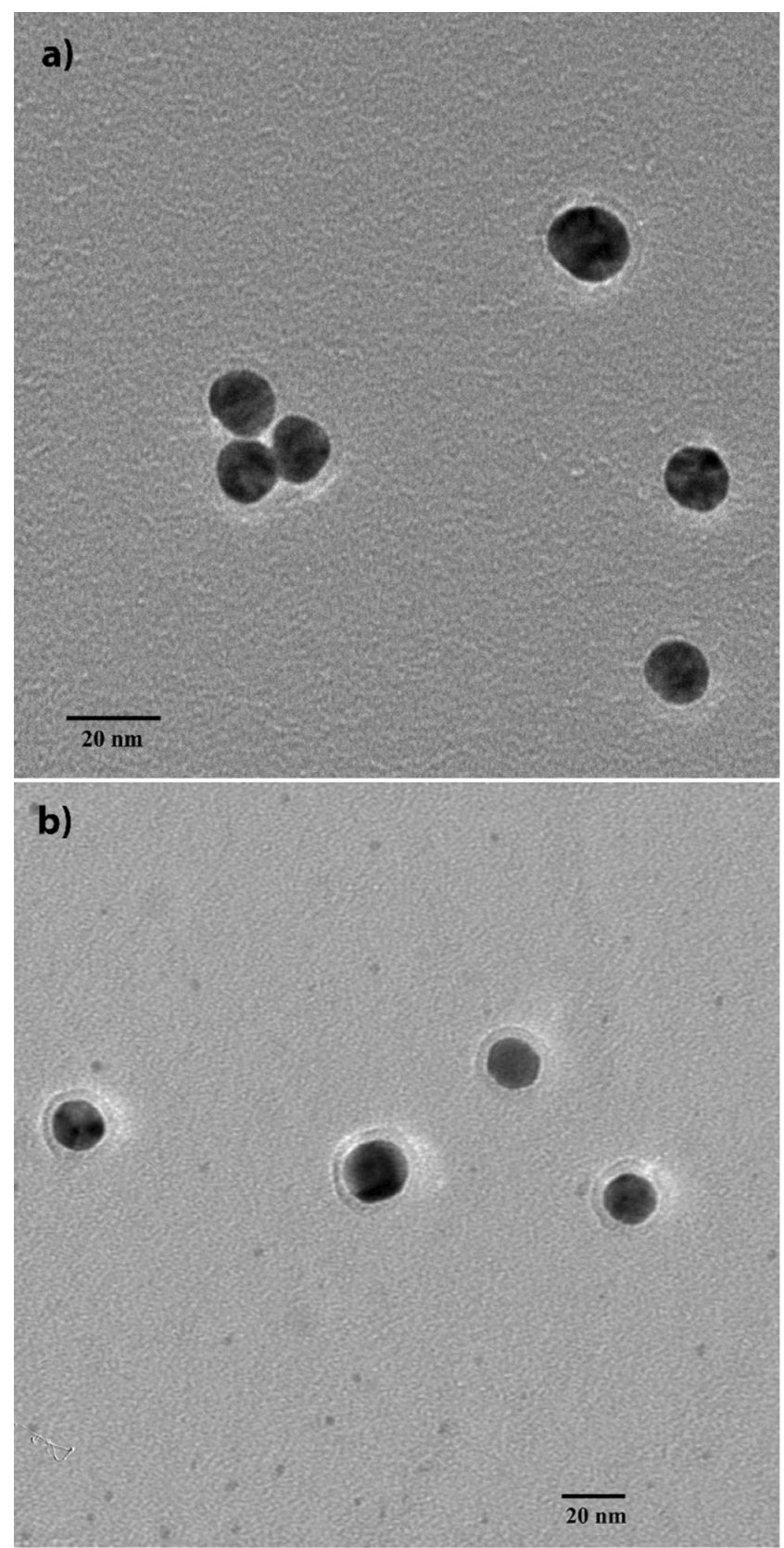

Fig. 3 TEM images of a $\mathrm{Au}_{0} \mathrm{PAH}$ nanostructures and $\mathbf{b} \mathrm{Au}_{2} \mathrm{PAH}$ nanostructures

opposed to what was observed for the biotinylation of the $\mathrm{Au}_{0} \mathrm{PAH}$ sample, in which the PE monolayer was not capable of preventing the formation of aggregates.

Fluorescent $\mathrm{Au} / \mathrm{PE}$ assemblies were prepared by labelling the outermost PE layers with a fluorescent organic dye. For that purpose, the PAH chains were first functionalized with FITC to afford PAHF which was then used in the coating of the $\mathrm{Au}$ NPs yielding FITC labelled $\mathrm{Au}_{0}$ PAHF and $\mathrm{Au}_{2} \mathrm{PAHF}$ assemblies. Similarly to the FITC non-labelled systems described above, these fluorescent nanostructures were used as starting colloids in biotinylation procedures. As expected, the FITC labelled $\mathrm{Au} / \mathrm{PE}$ nanostructures showed a broader 
Fig. 4 Visible spectra of $\mathrm{Au}_{0} \mathrm{PAH}(\mathbf{a})$ and $\mathrm{Au}_{2} \mathrm{PAH}(\mathbf{b})$ nanostructures (the spectra were normalized at $450 \mathrm{~nm}$ )
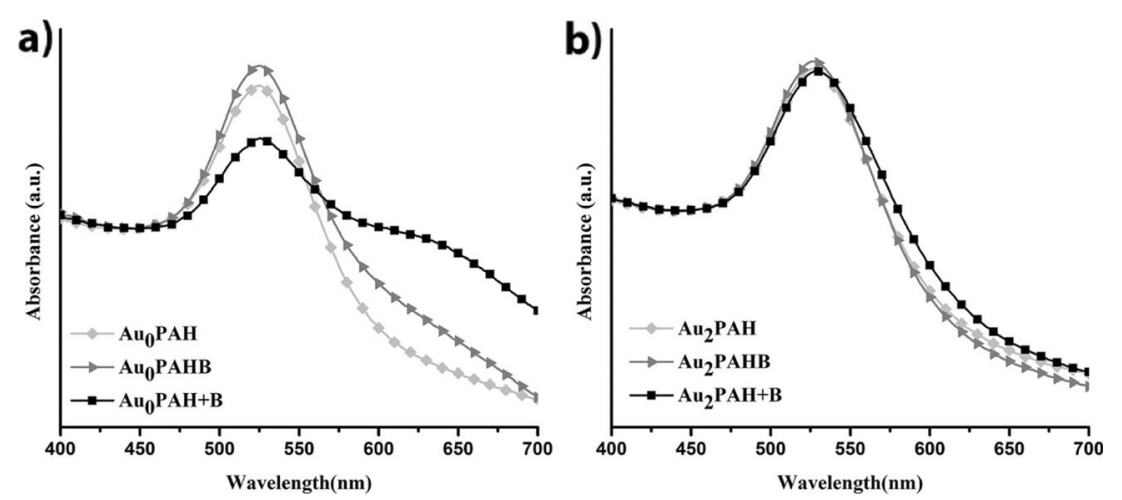

absorption band that takes into account the contribution of both the SPR band $\left(\lambda_{\max }=526.5 \mathrm{~nm}\right)$ of the Au NPs and the absorption due to FITC molecules $\left(\lambda_{\max }=497 \mathrm{~nm}\right)$. The visible spectra of biotinylated FITC labelled assemblies were similar regardless of the biotinylation procedure used (Fig. 5). However, as previously observed for the FITC unlabelled analogues, post-biotinylation of the $\mathrm{Au}_{0} \mathrm{PAHF}$ nanostructures caused significant changes on the respective visible spectrum, which is ascribed to the loss of colloidal stability during the biotinylation step. However, this was not observed for the $\mathrm{Au}_{2} \mathrm{PAHF}+\mathrm{B}$ assemblies. These results confirm that the presence of an extra PE bilayer leads to more robust colloids, thereby providing enhanced colloidal stability during post-biotinylation (Fig. 5a, c).

The fluorescence emission measurements (Fig. 5b, d) are in agreement with the results discussed above regarding the stability of each colloidal system. The fluorescence emission intensity depends on the biotinylation path followed and on the presence of the extra PE bilayer. Regarding the Au nanostructures with the extra PE bilayer, the presence of biotin has an incremental effect on the fluorescence emission intensity. For $\mathrm{Au}_{2} \mathrm{PAHFB}$, the increase of the fluorescence intensity is more pronounced when compared to the sample obtained via post-biotinylation (sample $\mathrm{Au}_{2} \mathrm{PAHF}+\mathrm{B}$ ). This is attributed to the fact that in sample $\mathrm{Au}_{2} \mathrm{PAHFB}$, the biotinylated polyelectrolyte may adopt a conformation around the Au NP that provides a longer distance between the fluorophore molecules and the metal surface, when compared to the non-biotinylated sample. In this situation, the fluorophore molecules being further away from the Au surfaces limit the FITC fluorescence quenching as a result of the fact that the process of energy transfer from FTIC (the donor) to Au (the acceptor) is dependent on the distance between them [35]. This explanation is in line with the observation that the $\mathrm{Au}_{2}$ PAHFB assemblies are
Fig. 5 a and $\mathbf{c}$ Visible spectra and $\mathbf{b}$ and $\mathbf{d}$ Fluorescence spectra $\left(\lambda_{\mathrm{exc}}=494 \mathrm{~nm}\right)$ of $\mathrm{Au}_{0} \mathrm{PAHF}$ and $\mathrm{Au}_{2}$ PAHF nanostructures. The visible spectra were normalized at $450 \mathrm{~nm}$
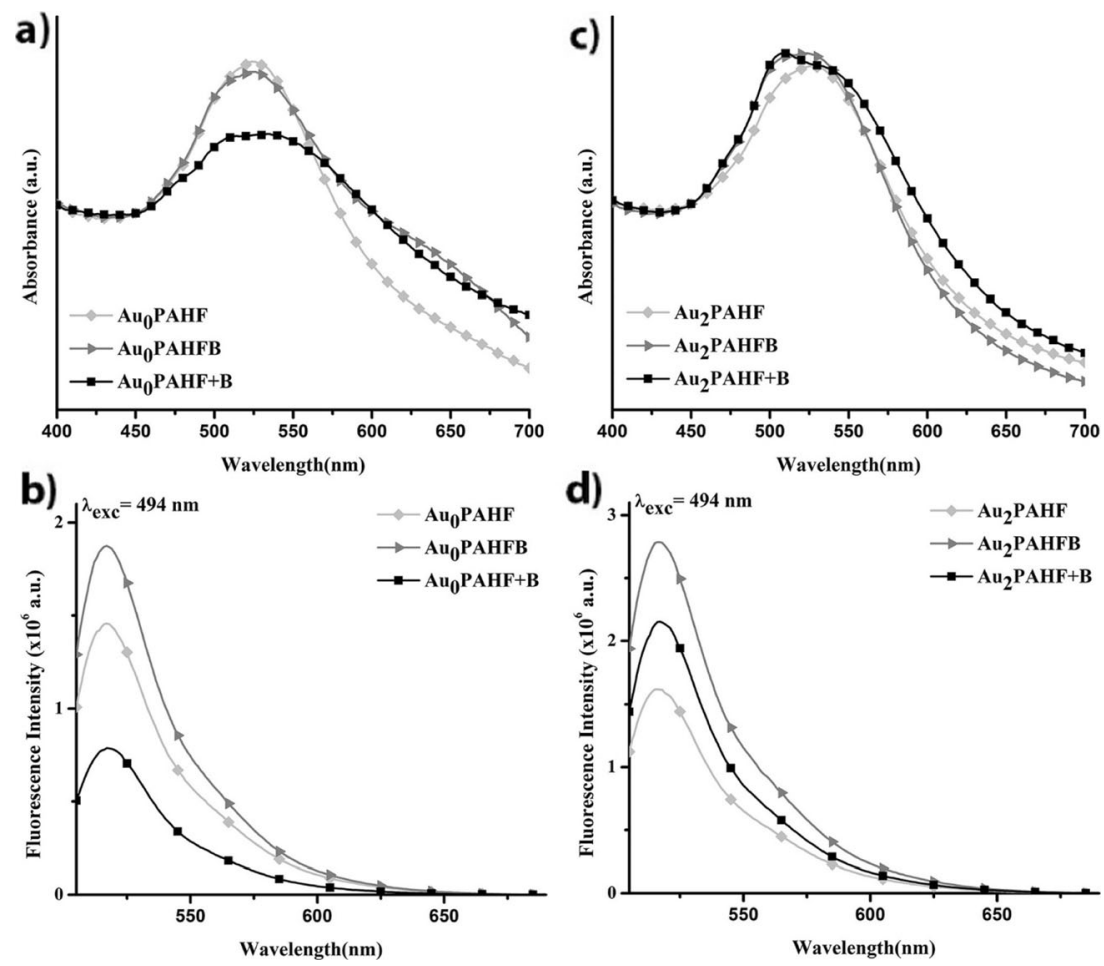
Fig. $6 \Delta A$ (avidin/colloid) of a $\mathrm{Au}_{0} \mathrm{PAH}$ and $\mathbf{b} \mathrm{Au}_{2} \mathrm{PAH}$ nanostructures in the presence of avidin
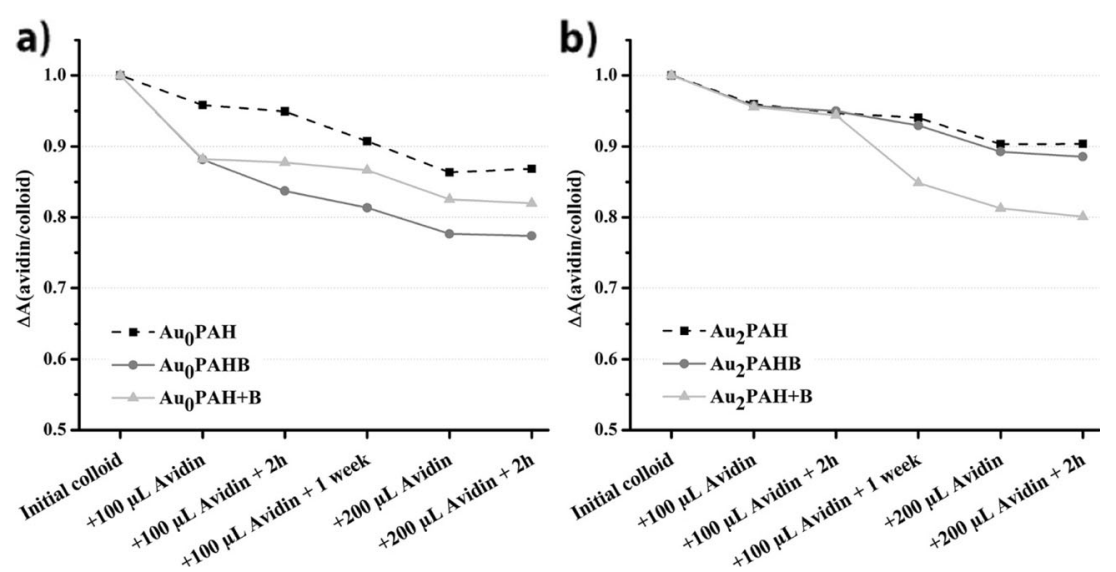

more stable with subsequent increase of the FITC fluorescence emission (Fig. 5d). As regards the sample $\mathrm{Au}_{2} \mathrm{PAHF}+$ $\mathrm{B}$, the increment on the fluorescence intensity was not so pronounced after biotinylation. This may be associated with the reduction of the number of protonated aminic groups available for colloidal electrostatic stabilization. As a result, the colloidal stability of the nanostructures is compromised causing a detrimental effect on the FITC fluorescence emission due to the reduced distance between the fluorophore and the Au surfaces. Note that this explanation is consistent with the visible spectrum of $\mathrm{Au}$ NPs modified with a single PE layer shown in Fig. 5a for the same sample. Indeed, the fluorescent intensity for the $\mathrm{Au}_{0} \mathrm{PAHF}+\mathrm{B}$ sample is even smaller than that recorded for the sample without biotin $\left(\mathrm{Au}_{0} \mathrm{PAHF}\right)$ as shown in Fig. 5b. However, when an extra PE bilayer was used, the detrimental effect of the postbiotinylation process was less pronounced.

In order to assess the optical response of the biotinylated $\mathrm{Au} / \mathrm{PE}$ nanostructures for biosensing, avidin was added to all of the nanostructures prepared including the Au nanostructures without the extra PE bilayer. The nanostructures without biotin were used as blanks regarding the response to avidin under the same experimental conditions. Then the effect of time and analyte concentration was assessed by exposing the
Fig. 7 a and c $\Delta A$ (avidin/ colloid) and $\mathbf{b}$ and $\mathbf{d} I_{517}$ (avidin)/ $I_{517}$ (colloid) of $\mathrm{Au}_{0} \mathrm{PAH}$ and $\mathrm{Au}_{2} \mathrm{PAHF}$ nanostructures in the presence of avidin, respectively
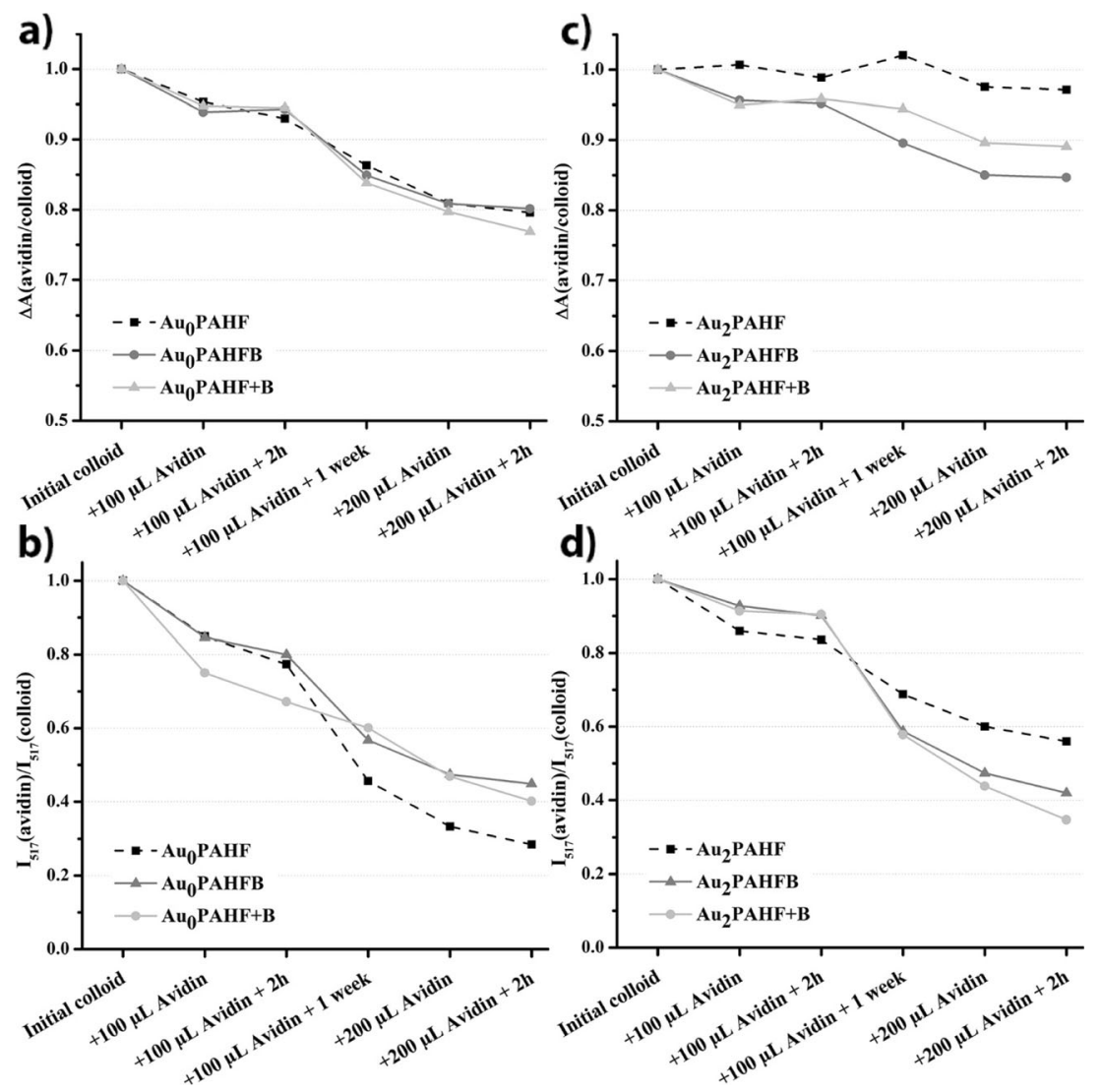
samples to solutions of avidin of two distinct concentrations and for different periods of time. All the results have been compiled and plotted in Figs. 6 and 7 as described in the "Materials and methods" section.

As regards the optical response of these assemblies towards the presence of avidin in terms of the absorbance of the SPR band, a general trend towards its reduction is observed as illustrated in Figs. 6 and 7a, c, which suggests that all the nanostructures respond to the presence of avidin, including those without biotin. This is an indication that both specific and non-specific interactions between avidin and the functionalized $\mathrm{Au} / \mathrm{PE}$ assemblies took place. The reason for non-specific interactions seems to be associated with changes of the surrounding medium that affects colloidal stability which is particularly evident for the non-labelled assemblies submitted to the post-biotinylation method (namely those prepared with only one layer of PAH—see Fig. 6a). Indeed, Qian et al. have reported that non-specific interactions of streptavidin with uncoated Au NPs occurred via uncertain group binding [42]. Therefore, these nonspecific interactions may result from deficient surface protection associated with changes of composition of the surrounding medium. In view of the susceptibility of the $\mathrm{Au}$ assemblies prepared with only one PE layer $\left(\mathrm{Au}_{0} \mathrm{NPs}\right)$, it is difficult to ascertain the formation of any specific interactions; thus, focus was given to the more robust assemblies prepared using the extra $\mathrm{PE}$ bilayer $\left(\mathrm{Au}_{2} \mathrm{NPs}\right)$. As regards the biotinylated $\mathrm{Au}$ nanostructures, only the $\mathrm{Au}_{2} \mathrm{PAH}+\mathrm{B}$ seem to show a distinct response towards the presence of avidin (Fig. 6b), whilst the samples labelled with FITC, i.e., $\mathrm{Au}_{2} \mathrm{PAHFB}$ and $\mathrm{Au}_{2} \mathrm{PAHF}+\mathrm{B}$, have both shown specific response when compared to the control sample $\mathrm{Au}_{2} \mathrm{PAHF}$ (Fig. 7c). In fact, when $100 \mu \mathrm{L}$ of avidin were used, the reduction of $\Delta A$ (avidin/colloid) was only noticeable upon 1 week. Yet, when $200 \mu \mathrm{L}$ of avidin were used, that reduction was almost immediate.

Whilst the optical spectra provide some information regarding the behavior of this type of $\mathrm{Au}$ assemblies towards the presence of avidin, more expressive results were obtained when fluorescence spectroscopy was used (Fig. 7d), which may indicate specific interaction between the biotin and the avidin. Although the observed decrease in the $I_{517}$ (avidin) $/ I_{517}$ (colloid) might be caused by particle aggregation following the addition of avidin, the optical response for the biotinylated samples was more pronounced which might be associated with their specific molecular recognition for avidin and associated quenching effects. Moreover, comparison with the results shown in Fig. 7b for the FITC labelled samples prepared with one single layer, it is clear that the effect of non-specific interactions leads to a distinct response associated with their poor colloidal stability but that cannot be discerned by optical spectroscopy.

\section{Conclusions}

Surface functionalization of gold nanoparticles was accomplished using functionalized polyelectrolytes and the electrostatic self-assembly method. The use of an extra PE bilayer between the $\mathrm{Au}$ core and the outermost functional layer proved to be crucial to provide robustness and stability to the ensuing nanostructures. Strategies to functionalize PAH led to the preparation of multifunctional Au nanostructures with fluorescence and biorecognition properties, which combine an organic fluorophore (FITC) and a biolinker (biotin). The functionalized Au nanostructures have shown potential for biosensing applications due to their optical response in forming biotin-avidin conjugates. Worth noting is the fact that in these tests, the analyte was not labelled with a fluorophore, as reported by others [41]. Moreover, whilst small changes due to particle aggregation during the bioassays caused a small reduction of $\Delta A$ (avidin/colloid), similar changes caused a pronounced decrease of the FITC emission band in the fluorescence emission spectra $\left(I_{517}\right.$ (avidin) $/ I_{517}$ (colloid)). Thus, fluorescence emission spectroscopy emerged here as an interesting tool to assess the ability of biotinylated $\mathrm{Au}$ assemblies to recognize avidin.

Acknowledgments This work was funded by Fundos FEDER by the Programa Operacional Factores de Competitividade - COMPETE and Fundos Nacionais by FCT-Fundação para a Ciência e Tecnologia in the project CICECO - FCOMP-01-0124-FEDER-037271 (Ref ${ }^{\mathrm{a}}$. FCT PEst-C/CTM/LA0011/2013). S. O. Pereira thanks FCT for her PhD grant SFRH/BD/80156/2011.

Open Access This article is distributed under the terms of the Creative Commons Attribution License which permits any use, distribution, and reproduction in any medium, provided the original author(s) and the source are credited.

\section{References}

1. Trindade T, da Silva ALD (eds) (2011) Nanocomposite particles for bio-applications. Materials and bio-interfaces. Pan Stanford Publishing Pte. Ltd., Singapore. doi:10.4032/9789814267816

2. Vidotti M, Carvalhal RF, Mendes RK, Ferreira DCM, Kubota LT (2011) Biosensors based on gold nanostructures. J Braz Chem Soc 22:3-20. doi:10.1590/S0103-50532011000100002

3. Zeng S, Yong K-T, Roy I, Dinh X-Q, Yu X, Luan F (2011) A review on functionalized gold nanoparticles for biosensing applications. Plasmonics 6:491-506. doi:10.1007/s11468-011-9228-1

4. Liz-Marzán LM (2004) Nanometals: formation and color. Mater Today 7:26-31. doi:10.1016/S1369-7021(04)00080-X

5. Dreaden EC, Alkilany AM, Huang X, Murphy CJ, El-Sayed MA (2012) The golden age: gold nanoparticles for biomedicine. Chem Soc Rev 41:2740-2779. doi:10.1039/c1cs15237h

6. Daniel M-C, Astruc D (2004) Gold nanoparticles: assembly, supramolecular chemistry, quantum-size-related properties, and 
applications toward biology, catalysis, and nanotechnology. Chem Rev 104:293-346. doi:10.1021/cr030698+

7. Saha K, Agasti SS, Kim C, Li XN, Rotello VM (2012) Gold nanoparticles in chemical and biological sensing. Chem Rev 112:27392779. doi:10.1021/cr2001178

8. Sato K, Hosokawa K, Maeda M (2003) Rapid aggregation of gold nanoparticles induced by non-cross-linking DNA hybridization. J Am Chem Soc 125:8102-8103. doi:10.1021/ja034876s

9. Storhoff JJ, Lazarides AA, Mucic RC, Mirkin CA, Letsinger RL, Schatz GC (2000) What controls the optical properties of DNAlinked gold nanoparticle assemblies? J Am Chem Soc 122:4640 4650. doi:10.1021/ja9938251

10. Kohut A, Voronov A, Peukert W (2005) Organization of functionalized gold nanoparticles by controlled protein interactions. Part Part Syst Charact 22:329-335. doi:10.1002/ppsc.200500986

11. Aslan K, Luhrs CC, Perez-Luna VH (2004) Controlled and reversible aggregation of biotinylated gold nanoparticles with streptavidin. J Phys Chem B 108:15631-15639. doi:10.1021/jp036089n

12. Turkevich J, Stevenson PC, Hillier J (1951) A study of the nucleation and growth processes in the synthesis of colloidal gold. Discuss Faraday Soc 11:55-75. doi:10.1039/DF9511100055

13. Gittins DI, Caruso F (2001) Tailoring the polyelectrolyte coating of metal nanoparticles. J Phys Chem B 105:6846-6852. doi:10.1021/ jp0111665

14. Gittins DI, Caruso F (2000) Multilayered polymer nanocapsules derived from gold nanoparticle templates. Adv Mater 12:19471949. doi:10.1002/1521-4095(200012)12:24<1947::aidadma1947>3.0.co;2-8

15. Higashi N, Takagi T, Koga T (2010) Layer-by-layer fabrication of well-packed gold nanoparticle assemblies guided by a [beta]-sheet peptide network. Polym J (Tokyo, Jpn) 42:95-99. doi:10.1038/pj. 2009.311

16. Cho JH, Caruso F (2005) Investigation of the interactions between ligand-stabilized gold nanoparticles and polyelectrolyte multilayer films. Chem Mater 17:4547-4553. doi:10.1021/cm050972b

17. Labouta HI, Schneider M (2010) Tailor-made biofunctionalized nanoparticles using layer-by-layer technology. Int J Pharm 395: 236-242. doi:10.1016/j.ijpharm.2010.05.019

18. Parveen S, Misra R, Sahoo SK (2012) Nanoparticles: a boon to drug delivery, therapeutics, diagnostics and imaging. Nanomedicine Nanotechnol Biol Med 8:147-166. doi:10.1016/j.nano.2011.05.016

19. Yeh Y-C, Creran B, Rotello VM (2012) Gold nanoparticles: preparation, properties, and applications in bionanotechnology. Nanoscale 4:1871-1880. doi:10.1039/C1NR11188D

20. Kimura M, Kobayashi S, Kuroda T, Hanabusa K, Shirai H (2004) Assembly of gold nanoparticles into fibrous aggregates using thiolterminated gelators. Adv Mater 16:335-338. doi:10.1002/adma. 200305804

21. Pinto RJB, Marques PAAP, Martins MA, Neto CP, Trindade T (2007) Electrostatic assembly and growth of gold nanoparticles in cellulosic fibres. J Colloid Interface Sci 312:506-512. doi:10.1016/j.jcis.2007. 03.043

22. Manson J, Kumar D, Meenan B, Dixon D (2011) Polyethylene glycol functionalized gold nanoparticles: the influence of capping density on stability in various media. Gold Bull 44:99-105. doi:10.1007/ s13404-011-0015-8

23. Caruso F, Caruso RA, Mohwald H (1998) Nanoengineering of inorganic and hybrid hollow spheres by colloidal templating. Science 282:1111-1114. doi:10.1126/science.282.5391.1111

24. Schneider G, Decher G (2008) Functional core/shell nanoparticles via layer-by-layer sssembly. Investigation of the experimental parameters for controlling particle aggregation and for enhancing dispersion stability. Langmuir 24:1778-1789. doi:10.1021/la7021837
25. Schonhoff M (2003) Self-assembled polyelectrolyte multilayers. Curr Opin Colloid Interface Sci 8:86-95. doi:10.1016/s13590294(03)00003-7

26. Schneider G, Subr V, Ulbrich K, Decher G (2009) Multifunctional cytotoxic stealth nanoparticles. A model approach with potential for cancer therapy. Nano Lett 9:636-642. doi:10.1021/n1802990w

27. Pereira SO, Barros-Timmons A, Trindade T (2013) Biofunctionalisation of colloidal gold nanoparticles via polyelectrolytes assemblies. Colloid Polym Sci 292:33-50. doi:10.1007/ s00396-013-3037-3

28. Deshmukh PK, Ramani KP, Singh SS, Tekade AR, Chatap VK, Patil GB, Bari SB (2013) Stimuli-sensitive layer-by-layer (LbL) selfassembly systems: targeting and biosensory applications. J Control Release 166:294-306. doi:10.1016/j.jconrel.2012.12.033

29. Schneider G, Decher G (2004) From functional core/shell nanoparticles prepared via layer-by-layer deposition to empty nanospheres. Nano Lett 4:1833-1839. doi:10.1021/n10490826

30. Dulkeith E, Morteani AC, Niedereichholz T, Klar TA, Feldmann J, Levi SA, van Veggel F, Reinhoudt DN, Moller M, Gittins DI (2002) Fluorescence quenching of dye molecules near gold nanoparticles: radiative and nonradiative effects. Phys Rev Lett 89:203002. doi:10. 1103/PhysRevLett.89.203002

31. Gueroui Z, Libchaber A (2004) Single-molecule measurements of gold-quenched quantum dots. Phys Rev Lett 93:1-4. doi:10.1103/ PhysRevLett.93.166108

32. Yun CS, Javier A, Jennings T, Fisher M, Hira S, Peterson S, Hopkins B, Reich NO, Strouse GF (2005) Nanometal surface energy transfer in optical rulers, breaking the FRET barrier. J Am Chem Soc 127: 3115-3119. doi:10.1021/ja043940i

33. Jennings TL, Singh MP, Strouse GF (2006) Fluorescent lifetime quenching near $d=1.5 \mathrm{~nm}$ gold nanoparticles: probing NSET validity. J Am Chem Soc 128:5462-5467. doi:10.1021/ja0583665

34. Sapsford KE, Berti L, Medintz IL (2006) Materials for fluorescence resonance energy transfer analysis: beyond traditional donor-acceptor combinations. Angew Chem Int Ed 45:4562-4588. doi:10.1002/anie. 200503873

35. Schneider G, Decher G, Nerambourg N, Praho R, Werts MHV, Blanchard-Desce M (2006) Distance-dependent fluorescence quenching on gold nanoparticles ensheathed with layer-by-layer assembled polyelectrolytes. Nano Lett 6:530-536. doi:10.1021/n1052441s

36. Dubertret B, Calame M, Libchaber AJ (2001) Single-mismatch detection using gold-quenched fluorescent oligonucleotides. Nat Biotechnol 19:365-370. doi:10.1038/86762

37. Maxwell DJ, Taylor JR, Nie SM (2002) Self-assembled nanoparticle probes for recognition and detection of biomolecules. J Am Chem Soc 124:9606-9612. doi:10.1021/ja025814p

38. Kato N, Caruso F (2005) Homogeneous, competitive fluorescence quenching immunoassay based on gold nanoparticle/polyelectrolyte coated latex particles. J Phys Chem B 109:19604-19612. doi:10. 1021/jp052748f

39. Park S, Lee S, Kim G, Kim Y-P (2012) Gold nanoparticle-based fluorescence quenching via metal coordination for assaying protease activity. Gold Bull 45:213-219. doi:10.1007/s13404-012-0070-9

40. Aslan K, Perez-Luna VH (2004) Quenched emission of fluorescence by ligand functionalized gold nanoparticles. J Fluoresc 14:401-405. doi:10.1023/B:JOFL.0000031821.74706.ea

41. Aslan K, Perez-Luna VH (2006) Nonradiative interactions between biotin-functionalized gold nanoparticles and fluorophore-labeled antibiotin. Plasmonics 1:111-119. doi:10.1007/s11468-006-9013-8

42. Li X, Jiang L, Zhan QQ, Qian J, He SL (2009) Localized surface plasmon resonance (LSPR) of polyelectrolyte-functionalized goldnanoparticles for bio-sensing. Colloids Surf A 332:172-179. doi:10. 1016/j.colsurfa.2008.09.009 